\title{
Comparing Factors influencing Heavy Episodic Drinking of Young Adults in the United Kingdom and the Netherlands
}

Short title: Heavy Episodic Drinking in UK and Dutch Youths

Sil Liebrand ${ }^{1}$, Judith Eberhardt², Paul van Schaik ${ }^{2}$, Marcel Pieterse ${ }^{1}$, Anna van Wersch ${ }^{2}$, Peter ten Klooster ${ }^{4}$, Henk Boer ${ }^{1}$

${ }^{1}$ University of Twente, Netherlands

${ }^{2}$ Teesside University, United Kingdom

${ }^{*}$ Corresponding author information: Judith Eberhardt, School of Social Sciences, Humanities and Law, Teesside University, Borough Road, Middlesbrough TS1 3BA, United Kingdom. E-mail: j.eberhardt@tees.ac.uk. ORCiD: https://orcid.org/00000003-0745-178X 


\begin{abstract}
The United Kingdom and the Netherlands exhibit similar levels of heavy episodic drinking but different drinking patterns among youths. This study aimed to assess the impact of country of residence on heavy episodic drinking among 293 British and Dutch youths, accounting for other behavioral determinants. Participants completed online questionnaires measuring impulsivity, sensation-seeking, alcohol consumption, and constructs from the Theory of Planned Behavior [TPB]. Mediation analysis established that British youths engaged in more frequent heavy drinking episodes than Dutch youths, as they had weaker intentions to refrain from heavy drinking, and lower perceived behavioral control. Country of residence also was a direct predictor of frequency of heavy drinking episodes, not mediated by personality traits. Thus, country of residence seems an important factor in heavy episodic drinking, partly mediated through TPB constructs. Interventions may benefit from targeting country-specific drinking behavior and related socio-psychological mechanisms.
\end{abstract}

Keywords: Heavy episodic drinking; theory of planned behavior; sensation-seeking; 'dry' versus 'wet' country comparison

\title{
Introduction
}

Heavy episodic drinking (HED) has short- and long-term major consequences for public health and society (van Wersch \& Walker, 2009; World Health Organization [WHO], 2011) and is highly prevalent around the world (Fuller-Thomson et al., 2013). The population of the European Union (EU) has been shown to have the highest alcohol intake (Anderson \& Baumberg, 2006). In this research, HED was defined as 
drinking 'at least six units of alcohol during one sitting' (e.g. Garretsen, Rodenburg, van de Goor \& van den Eijnden, 2008). The United Kingdom (UK) and the Netherlands are both at the top of the list of EU countries with the highest prevalence of HED amongst students. Hibell et al. (2004) noted that in 2003, 54\% of English students and $58 \%$ of Dutch students engaged in HED; similar rates were observed in 2007 (Hibell et al., 2009). These figures stand in contrast to other European countries such as France (28\%), Portugal (25\%), and Romania (23\%).

While the aggregated amount of HED amongst students appears comparable between the UK and the Netherlands, there seem to be some significant variances in specific drinking patterns which could be due to country-specific differences (Gordon, Heim \& MacAskill, 2012). The prevailing drinking pattern in the UK has been described as a 'dry culture', as opposed to a predominantly 'wet culture' in The Netherlands. In a 'dry' drinking culture, people drink little or nothing during the week, but go out and drink heavily over the weekend, with the main intention of getting drunk (Gordon et al., 2012). Going out to drink is typically carefully planned amongst a group of people to ensure their safety when intoxicated. They rarely drink and drive and remain sober when they have important activities the next day, for example work (Szmigin et al., 2008; van Wersch \& Walker, 2009). This calculated hedonistic style seems to be the norm in the UK and contributes to the onset of HED at an early age (Szmigin et al., 2008). The Licensing Act (2003) which came into effect in 2005 extended the opening hours of pubs, which was expected to lead to a reduction of rapid intake of alcohol to 'beat the clock'. However, there is evidence to indicate that this policy change may in fact have exacerbated the problem, at least for those who already drank heavily (Green, Hollingsworth \& Navarro Paniagua, 2015). 
Conversely to 'dry' countries, in 'wet' countries people highly value the ability to hold their liquor, drinking moderately but more frequently (Gordon et al., 2012). Moderate drinking while going out seems to be the norm in the Netherlands, even in young adults (Hughes et al., 2011). Presently, the Dutch legislation states that the legal drinking age is eighteen, but at the time of the study the legal drinking age was still sixteen. Furthermore, serving alcohol is allowed when an alcohol-serving licenseholding place is open (Overheid.nl, 2013). This might influence people to drink more steadily over the day, thus limiting alcohol intoxication.

In both countries regional differences in HED among youth appear to exist. In particular the north-east of England seems to be an area where HED is relatively common. Binge drinking is the most prevalent in this region (Office for National Statistics, 2016) and hospital admission episodes for alcohol-specific conditions among individuals under 18 years are significantly above the UK average (Local Alcohol Profiles for England, 2017). In the Netherlands, the East also seems to strongly contribute to the country's total alcohol consumption (De Staat van Volksgezondheid en Zorg, 2018; Volksgezondheidenzorg.info, 2018). In terms of addressing HED as a public health issue, young people are an important target group, since intervening early on may help prevent the development of alcoholrelated problems later in life as well as other risky behaviors such as crime, smoking and cannabis use (Green \& Ross, 2010). 
The Theory of Planned Behavior (TPB; Ajzen, 1991) has been applied extensively in binge-drinking research. It posits three motivational factors that influence the intention to perform certain behaviors, namely attitudes, subjective norms and perceived behavioral control. Attitudes refer to the evaluation people make of their own behaviors. Research has shown that there are two main positive attitudes towards alcohol: pleasure and relaxation (Kuntsche, Rehm \& Gmel, 2004), which have been found to predict future drinking quantity and frequency in North American college students (Collins \& Carey, 2007). Positive attitudes towards binge drinking have been found, among other variables, to be associated with strong intentions to engage in binge drinking in the near future in UK undergraduate students (Norman, Conner \& Stride, 2012). Subjective norm implies a person's perception of others' evaluation of the person's behavior. Perceived behavioral control (PBC) refers to the perceived level of difficulty for a specific person to execute certain behavior, such as refusing alcoholic drinks, which has been found to be a strong predictor of quantity and frequency of alcohol use and problems related to drinking (Collins \& Carey, 2007). In a British study, Norman and Conner (2006) concluded that low PBC was associated with strong alcohol-use intentions in students. Similarly, Norman, Armitage and Quigley (2007) reported that UK undergraduates who were low in PBC regarding bingedrinking were more likely to engage in this behavior over the following week. Marks Woolfson and Maguire (2010) found similar results in Scottish students. However, another UK study by French and Cooke (2012) failed to detect any association between PBC and intention to binge drink; the authors suggest that this could be attributable to a lack of internal consistency of the measure of 
PBC used, as well as a 'ceiling effect' resulting from measuring PBC in students who were currently at a bar. A meta-analysis of 40 studies, the majority of which were British, supported the utility of the TPB when applied to alcohol consumption intentions and behavior, with medium-to large effect sizes being found for many of the TPB relationships (Cooke, Dahdah, Norman, \& French, 2016).

While these findings may reflect a connection between PBC and intention to drink excessively in young British people, there is a lack of cross-country comparison regarding PBC or other variables of the TPB. The similar levels of alcohol consumption among UK and Dutch young individuals and the fact that PBC appears to be such a strong predictor of alcohol consumption patterns, highlight the need for examining potential differences in PBC between these two groups as well as the relationship with both the intention to binge drink and actual drinking behavior. Such a comparison would be useful because, although rates of consumption are similar in the two populations, patterns of consumption differ, with heavy episodic drinking more prevalent in the UK population.

In addition to constructs contained within the TPB, personality traits have been found to play a role. Specifically, externalizing traits - impulsivity (IMP) and sensation-seeking (SESE) - have been implicated in HED (Stautz \& Cooper, 2013). Impulsivity seems to be associated with risky behaviors such as alcohol use due to poor motor and cognitive inhibition (López-Caneda et al., 2013). Sensation-seekers often have an urge for excitement and therefore are more 
open to excessive alcohol use. The association between HED, measured by self-reported alcohol consumption patterns and number of drinks consumed in one session, with impulsivity and inability to inhibit a response was established by Henges and Marczinski (2012); similar results have been found in other studies (e.g. Carlson, Johnson \& Jacobs, 2010; Lynam \& Miller, 2004). Furthermore, greater amounts of alcohol tend to be consumed per drinking session by those higher in impulsivity and sensation-seeking (Reich, Cummings, Greenbaum, Moltisanti, \& Goldman, 2015). This established link between HED and impulsivity-sensation-seeking has not yet been examined across countries. Doing so would help shed light on the country-specific differences between the UK and the Netherlands regarding HED. In a 'dry' culture, giving in to the impulse of binging may be seen as more acceptable than in a 'wet' culture. As a result, individuals with high trait impulsivity would likely be less motivated to control HED. Consequently, impulsivity would be more strongly associated with HED within a 'dry' culture.

To summarize, it would be useful to examine variables of the TPB, especially PBC, as well as impulsivity and sensation-seeking, and their relationship to HED, in both UK and Dutch young people, in light of their similar levels but differing patterns of alcohol consumption. A cross-country comparison of these factors would help understand why UK youths consume alcohol differently from Dutch youths, thus enabling interventions to be tailored to the target group.

Accordingly, the main aims of the study were to 1) determine how country of residence impacts on determinants associated with HED among young adults, and 
2) identify and compare country-specific differences in mechanisms underlying HED among young adults in the UK and the Netherlands. A further aim was to explore the role of impulsivity and sensation-seeking.

\section{Materials and Methods}

\section{Research Design and Participants}

A cross-sectional survey design was used. There were 293 participants with a mean age of $18.87(S D=2.11)$, an age range of 15 to 25 , and a $65 / 35$ gender split (190 female/103 male); 198 were Dutch and 95 British. A convenience sampling method was used, with individuals being approached on the street as well as the study being advertised online to students and on social media. Ethics approval for this research was granted by the research ethics committee of the lead author's institution.

\section{Measurement}

The online questionnaire consisted of 43 questions and was developed by the research team in English and Dutch. Two personality traits were measured that are associated with substance use (Malmberg et al., 2012; Woicik, Stewart, Pihl, \& Conrod, 2009). These were two subscales of the Substance Use Risk Profile Scales [SURPS]: impulsivity (IMP), measured with four items (Cronbach's alpha $=0.59$; example items include 'I often involve myself in situations that I later regret being involved in', and 'I usually act without stopping to think'), and sensation-seeking (SESE), measured with five items (alpha $=0.62$; example items include ' $/$ enjoy new and exciting experiences even if they are unconventional', and 'I would like to skydive'). Participants were presented with item statements and asked to rate impulsive and sensation-seeking behaviors using a 4-point Likert scale: 'Strongly disagree', 'Disagree', 'Agree' and 'Strongly agree'. Principal component analysis 
showed that the items for the two traits loaded on two distinct factors without crossloadings (see Table A1).

[Table A1 near here]

For alcohol consumption, monthly frequency of heavy-drinking episodes (HDEs) was used, based on O’Malley, Bachman and Johnston (1983), and Korte, Pieterse, Postel \& Van Hoof (2012). First a definition of a unit of alcohol was provided, supported by a visualization of different types of drinks with corresponding units displayed in a table. This was adapted respectively to UK and Dutch drinking patterns, by showing pictures of common brands and beverages. HDE was then measured with the following question: 'How many times in the past 4 weeks did you have 6 or more units of alcohol on one occasion (e.g. at a party or on a night out)?'. Possible answers were I never drank more than 6 units in the past 4 weeks, 1, 2, 3, $4,5,6,7,8$ or 9 times or more in the past 4 weeks.

Two main constructs of the theory of planned behavior (Ajzen, 1991) were included. Behavioral intention was measured by three items (alpha $=0.84$; example items include '[For the next 6 months] I intend to drink less than 6 units of alcohol per occasion' and '[For the next 6 months] I intend never to exceed my 'tax' [so always drink less alcohol than your previously stated personal limit]'). Participants could answer by means of a 5-point scale; 'Definitely won't', 'Probably won't', 'Maybe', 'Probably will' and 'Definitely will'. Perceived behavioral control was measured by five items (alpha = 0.80; example items include 'To me, drinking less than 6 units of alcohol on one night, when my friends drink more is...' and 'To me, refusing an offered alcoholic drink when I actually have had enough is...') in which participants were asked to indicate their answers on a 5-point Likert scale: 'Easy', 'Relatively 
easy', 'Not easy, not hard', 'Relatively hard' and 'Hard'. High scores indicated high behavioral intention to refrain from HED and high perceived behavioral control over HED, respectively. Principal component analysis showed that the items for the two constructs loaded on two distinct factors without cross-loadings (see Table A2).

[Table A2 near here]

\section{Procedure}

The British cohort was recruited using convenience sampling methods from Teesside University and asked to cooperate in the online study. Participants received flyers with links to the online survey; a link was also posted on social media. Undergraduate psychology students were able to receive credits after finishing the survey, but $98 \%$ of the participants did not receive any compensation. Dutch participants were recruited in a similar way as the UK participants from the University of Twente. However, a monetary reward was offered in the form of $a € 10$ gift certificate raffle for completed surveys. An online questionnaire with all the measures as well as demographics was implemented. Participants were directed to the questionnaire via a web link. All participants gave informed consent to participate in the study, before filling in the questionnaire.

\section{Data Analysis}

Data were analyzed using descriptives, correlations and mediation analysis. All except two variables (heavy-drinking episodes and perceived behavioral control, which were skewed and kurtotic) followed a normal distribution. Transformations (square-root, logarithmic and inverse) were explored to improve the distributions. After transformations, the distributions were not normal; therefore the original untransformed variables were used. Thus, the true association between these and 
the remaining variables will be underestimated. Mediation analysis assumes a lack of (multi)collinearity. The variables did not suffer from collinearity (all absolute correlations $<0.60$ ) or multicollinearity (tolerance $>0.62$ for all).

\section{Results}

\section{Descriptives}

Heavy-drinking episodes (HDEs) were more frequent in British participants (medium effect size) (see Table 1). Dutch participants had a stronger intention and perceived behavioral control than British participants to refrain from heavy drinking (small to medium effect size). In terms of impulsivity and sensation-seeking, very small differences existed between British and Dutch participants.

[Table 1 near here]

\section{Correlations}

Behavioral intention was strongly negatively correlated with HDEs $(p<0.001)$, so the higher participants' intention to refrain from heavy drinking the less frequent their HDEs (see Table 2). Perceived behavioral control was strongly positively correlated with HDEs $(p<0.001)$, so the more difficult participants found it to refrain from heavy drinking, the more frequent their HDEs. Impulsivity and sensation-seeking were moderately correlated with HDEs (both $p<0.001$ ), so the more impulsive and the more sensation-seeking participants were, the more frequent their HDEs.

[Table 2 near here]

\section{Mediation Analysis}

Mediation analysis was conducted for two reasons. The first was to ascertain that established correlates of behavior (behavioral intention and perceived behavioral control) from the theory of planned behavior are mediators of the effect of nationality 
on HDEs. The second was to explore the role of established personality variables associated with HDEs (impulsivity and sensation-seeking) in the mediation. ${ }^{1}$

Consistent with the descriptives by country (see Table 1), mediation analysis showed country was significantly negatively associated with behavioral intention, but a significantly positively associated with perceived behavioral control (Figure 1). Also consistent with the descriptives, mediation analysis showed country was not significantly associated with either impulsivity or sensation-seeking.

Regarding HDEs, the positive direct effect of country, the negative effect of the mediator behavioral intention and the positive effects of the mediator perceived behavioral control and of impulsivity were significant. The positive effect of sensation-seeking was approaching significance. Mediation of the positive indirect effect of country by behavioral intention on HDEs was significant, as was mediation by perceived behavioral control. Mediation of the indirect effect by either impulsivity or sensation-seeking was not significant. The results of the mediation analysis showed that both the direct effect and the total indirect effect (over the four variables tested as mediators) of country on HDEs had a positive sign and are significant. Therefore, in terms of Zhao, Lynch and Chen's (2010) framework of mediation analysis, the patterns of results indicate complementary mediation. The results regarding the mediated indirect effect mean that British youths had more frequent HDEs than Dutch youths because their intention to refrain from heavy drinking and their perceived control over this were weaker. However, this can only be a partial

\footnotetext{
${ }^{1}$ Further mediation analysis was conducted to account for gender as a covariate. As the covariate was not significant, we do not report these results here. When male $(n=103)$ and female $(n=190)$ respondents were analysed separately, no significant indirect effect was found for males. For females, the indirect effect for intention was significant, as was the indirect effect of the combined mediators. We interpret the non-significant results as due to a loss of statistical power due to reduced sample size in the analysis by gender.
} 
explanation because the results of the non-mediated direct effect mean that potential additional mediators may exist that further explain why the two nations differed in HDEs.

[Figure 1 near here]

\section{Discussion}

The present study examined heavy episodic drinking (HED) behaviors of UK and Dutch young adults, focusing on the role of country of residence as well as comparing country-specific differences in causal mechanisms underlying these behaviors.

Differences were found regarding heavy drinking episodes (HDEs), with UK participants engaging more frequently in these than Dutch participants. Previous research found similar rates of HDEs for both groups, although Dutch participants tended to engage in more such episodes (Hibbell et al., 2004; 2009). Mediation analysis established a direct effect and an indirect effect of country on HDEs. The indirect effect showed that British youths engaged in more frequent HDEs than Dutch youths, which was explained by their weaker intentions to refrain from heavy drinking, and their weaker perceived control over their drinking. The more difficult participants found it to refrain from heavy drinking, the more frequent their HDEs; this relationship between perceived behavioral control and HDEs has been reported in previous British and American studies (Collins \& Carey, 2007; Norman et al., 2007; Marks Woolfson \& Maguire, 2010).

However, weaker intention and perceived behavioral control in British youths can only partially explain the differences in frequencies of HDEs between the two groups, because there was also a non-mediated direct effect of country. Therefore, potential 
additional mediators exist that account for the differences between the two groups. Further research would be necessary to explore such additional mediators.

In the current study, cross-national differences in HED could not be explained by differences in personality as a causal mechanism between countries, and this lack of mediation appeared to not depend on gender. The more impulsive and the more sensation-seeking participants were, the more frequent their HDEs, regardless of country of residence. The current literature points to personality variables as a potential source of influence on binge drinking. Attempts have been made to characterize binge drinkers in terms of their personality profiles. For example, in a study by Pirkle and Richter (2006), personality, attitudinal and behavioral correlates of binge drinking and smoking among adolescent girls and young women were identified and risk profiles of these behaviors generated. A Dutch study examined the mediation by drinking motives of the association between personality traits (including impulsivity and sensation-seeking) and alcohol frequency, binge drinking, and alcohol-related problems in adolescents (Lammers, Kuntsche, Engels, Wiers, \& Kleinjan, 2013). Differences were reported between males and females regarding motivational factors, which mediated the relationship between personality traits and binge drinking. However, neither study included cognitive and/or behavioral correlates. Moreover, neither study examined the role of personality as a mediator of the influence of country of residence on HED. To the authors' knowledge, the current study is the first to do so.

\section{Strengths, Limitations and Future Directions}

To the authors' knowledge, this is the first study to examine country of residence, social-cognitive variables and personality to more comprehensively analyse the 
determinants of HED. The results of the present study suggest that country of residence may be an important factor in HED, independently of other variables. Further investigation is necessary to determine why differences between countries play a role in HED. For example, it has been posited that the limited availability of leisurely activities for young people in the UK might contribute to HED as this has been suggested to increase boredom, which could lead to alcohol use (Biolcati, Passini, \& Mancini, 2016; McMahon, McAlaney, \& Edgar, 2007). Furthermore, there may be more leisure activities available for adolescents in the Netherlands - Dutch families with a low social economic status (SES) can apply for financial support so their children can join a sport club or play an instrument (Nibud, 2011), which has been shown to alleviate boredom, thus reducing alcohol consumption (Andersson, Andersson, Holmgren, Mårdby, \& Hensing, 2012).

Identifying how nationality plays a role in HED would help devise interventions designed to tackle this behavior.

It could be argued that the data for this study were collected in two specific regions of the UK and the Netherlands which limits generalizability. However, in these two regions, when compared to the other regions of their country, the prevalence of binge drinking was above the national average; therefore, a comparative analysis was appropriate.

The current study used self-report measures to elicit behavior. This may have resulted in inaccurate reporting of alcohol consumption, since response accuracy is influenced by the interaction of social context factors, respondent characteristics and task attributes (Del Boca \& Darkes, 2003). On the other hand, the data were collected over the Internet rather than face-to-face; there is evidence to indicate that 
measuring alcohol consumption in this way is more likely to result in disclosure than through other forms of measurement, such as paper-and-pencil questionnaires (Booth-Kewley, Larson, \& Miyoshi, 2007; Kays, Gathercoal, \& Buhrow, 2012). Consequently, the bias resulting from self-report is likely to be reduced in this study. Furthermore, as the majority of available studies in this field rely on self-reported data, using these type of data does not limit comparability with previous evidence. A difference in the recruitment procedure between the Dutch and UK sample, where only the Dutch participants were offered a small financial incentive, may have led to a sampling bias affecting our findings. The UK sample may have been more strongly selected on an intrinsic motivation to participate, which in turn may have resulted in relatively favorable alcohol cognitions and consumption estimates for this UK sample. In fact, it has been shown for alcohol use in adolescents that prevalence estimates of drinking are strongly underestimated when sampling is voluntary (i.e. more intrinsically motivated), as compared to mandatory (Cheung, ten Klooster, Smit, de Vries, \& Pieterse, 2017). More importantly, though, the study by Cheung et al. also showed that analyses of within-subject associations were insensitive to this sampling bias. Consequently, although the alcohol consumption level of our UK sample may well be underestimated (and more than in the Dutch sample), this is not likely to have confounded the correlational and mediational analyses reported here. Assessing true alcohol consumption levels was outside the scope of the current paper, which aimed solely at theory-based determinants of drinking behavior.

For both measures used for impulsivity and sensation-seeking, rather low internal consistency was found in this dataset. Although this is not uncommon in short scales (Loewenthal, 1996), and in previous studies using the SURPS Cronbach's Alphas of 
well below .70 have been reported for these two subscales (see f.e. Castellanos \& Conrod, 2006; Malmberg, 2010), this reliability issue may have confounded our findings. In particular, the absence of mediational effects of these traits as crossnationally specific mechanisms in this study may in part be the result of measurement error, rather than a true null finding.

The cross-sectional design of the present study prevents causal inferences from being made. However, it points to the need to explore the relationship between country, perceived behavioral control, impulsiveness and sensation-seeking, and heavy-drinking episodes in a way that allows causal connections to be examined. This could be achieved by conducting prospective studies, for example. Such studies could offer valuable insights for interventions aimed at heavy episodic drinking.

\section{Conclusion}

The results of this cross-country research comparing the UK to the Netherlands show that differences in countries' drinking patterns are an important correlate of HED, directly as well as indirectly, its effect being mediated by both TPB constructs and personality. Our findings highlight the importance of considering country-specific drinking patterns and their influence on HED. If the influence of drinking patterns on HED differs between countries, this implies that drink awareness campaigns and other HED-specific interventions and health promotion campaigns must be designed to take this into account. Health psychologists who are active in the area of health promotion or HED-related areas are ideally positioned to help raise awareness of the role of country-specific drinking patterns as well as lead on the design of such campaigns. However, the present results also support the roles of perceived behavioral control, behavioral intention, impulsivity and sensation seeking in HED among young adults. HED is a behavior jointly produced by psychosocial 
determinants at the situational, individual and environmental levels (Kuntsche, Kuntsche, Thrul, \& Gmel, 2017). If a deeper understanding is gained of how these factors collectively produce HED, individual risk profiles could be generated for heavy episodic drinkers, factoring in not only personality traits but also the influence of country-specific drinking patterns. This would allow for the design and implementation of culture-sensitive interventions for heavy episodic drinking in young adults.

\section{Declaration of Interest Statement}

The authors declare that they have no conflict of interest.

\section{References}

Ajzen, I. (1991). The theory of planned behavior. Organizational Behavior and Human Decision Processes, 50(2), 179-211. doi:10.1016/0749-5978(91)90020-T.

Anderson, P., \& Baumberg, B. (2006). Alcohol in Europe - public health perspective: Report summary. Drugs: Education, Prevention, and Policy, 13, 483-488. doi:10.1080/09687630600902477

Andersson, A., Andersson, C., Holmgren, K., Mårdby, A. C., \& Hensing, G. (2012).

Participation in leisure activities and binge drinking in adults: Findings from a Swedish general population sample. Addiction Research \& Theory, 20, 172-182. doi:10.3109/16066359.2011.596595

Biolcati, R., Passini, S., \& Mancini, G. (2016). "I cannot stand the boredom." Binge drinking expectancies in adolescence. Addictive Behaviors Reports, 3, 70 - 76. doi:10.1016/j.abrep.2016.05.001 
Booth-Kewley, S., Larson, G. E., \& Miyoshi, D. K. (2007). Social desirability effects on computerized and paper-and-pencil questionnaires. Computers in Human Behavior, 23, 463-477. doi:10.1016/j.chb.2004.10.020

Carlson, S. R., Johnson, S. C., \& Jacobs, P. C. (2010). Disinhibited characteristics and binge drinking among university student drinkers. Addictive Behaviors, 35, 242-251. doi:10.1016/j.addbeh.2009.10.020

Castellanos, N., \& Conrod, P. (2006). Brief interventions targeting personality risk factors for adolescent substance misuse reduce depression, panic and risk-taking behaviors. Journal of Mental Health, 15(6), 645 - 658.

doi:10.1080/09638230600998912

Cheung, K. L., Ten Klooster, P. M., Smit, C., \& Pieterse, M. E. (2017). The impact of nonresponse bias due to sampling in public health studies: A comparison of voluntary versus mandatory recruitment in a Dutch national survey on adolescent health. $B M C$ Public Health, 17(1), 276-10. doi:10.1186/s12889-017-4189-8

Collins, S. E., \& Carey, K. B. (2007). The theory of planned behavior as a model of heavy episodic drinking among college students. Psychology of Addictive Behaviors, 21, 498 - 507. doi: 10.1037/0893-164X.21.4.498

Cooke, R., Dahdah, M., Norman, P., \& French, D. P. (2016). How well does the theory of planned behavior predict alcohol consumption? A systematic review and metaanalysis. Health Psychology Review, 10(2), 148-167. doi:10.1080/17437199.2014.947547

De Staat van Volksgezondheid en Zorg (2018). Alcohol: overmatige drinkers [Alcohol: excessive drinkers]. Retrieved from https://www.staatvenz.nl/kerncijfers/alcoholgebruik-overmatige-drinkers 
Del Boca, F. K., \& Darkes, J. (2003). The validity of self-reports of alcohol consumption:

State of the science and challenges for research. Addiction, 98(S2), 1-12. doi:10.1046/j.1359-6357.2003.00586.x

French, D. P., \& Cooke, R. (2012). Using the theory of planned behaviour to understand binge drinking: the importance of beliefs for developing interventions._British Journal of Health Psychology, 17, 1-17. doi:10.1111/j.2044-8287.2010.02010.x

Fuller-Thomson, E., Sheridan, M. P., Sorichetti, C., \& Mehta, R. (2013). Underage binge drinking adolescents: Sociodemographic profile and utilization of family doctors. ISRN Family Medicine, 2013, 728730. doi: 10.5402/2013/728730

Garretsen, H. F. L., Rodenburg, G., van de Goor, L. A. M., \& van den Eijnden, R. J. J. M. (2008). Alcohol consumption in the Netherlands in the last decade: Sharp decreases in binge drinking, especially among youngsters. Alcohol \& Alcoholism, 4, 477 - 480. doi:10.1093/alcalc/agn037

Gordon, R., Heim, D., \& MacAskill, S. (2012). Rethinking drinking cultures: A review of drinking cultures and a reconstructed dimensional approach. Public Health, 126, 3 11. doi:10.1016/j.puhe.2011.09.014

Green, C. P., Hollingsworth, B. P., \& Navarro Paniagua, M. (2015). Longer opening hours, alcohol consumption and health. Working Paper. Lancaster University, Department of Economics, Lancaster.

Green, R. \& Ross, A. (2010). Young people's alcohol consumption and its relationship to other outcomes and behaviour. Department for Education Research Report. Retrieved from https://www.gov.uk/government/uploads/system/uploads/attachment_data/file/18243 2/DFE-RR005.pdf 
Henges, A. L., \& Marczinski, C. A. (2012). Impulsivity and alcohol consumption in young social drinkers. Addictive Behaviors, 37, 217-220. doi:10.1016/j.addbeh.2011.09.013

Hibell, B., Andersson, B., Bjarnason, T., Ahlström, S., Balakireva, O., Kokkevi, A., \& Morgan, M. (2004). The ESPAD Report 2003. Alcohol and other drug use among students in 35 European countries. Stockholm: Modintryckoffset AB.

Hibell, B., Guttormsson, U., Ahlström, S., Balakireva, O., Bjarnason, T., Kokkevi, A., \& Kraus, L. (2009). The 2007 ESPAD Report. Substance use among students in 35 European countries. Stockholm: Modintryckoffset AB.

Hughes, K., Quigg, Z., Bellis, M. A., van Hasselt, N., Calafat, A., Kosir, M., Juan, M., Duch, M., \& Voorham, L. (2011). Drinking behaviours and blood alcohol concentration in four European drinking environments: a cross-sectional study. BMC Public Health, 11, 1-11. doi:10.1186/1471-2458-11-918

Kays, K., Gathercoal, K., \& Buhrow, W. (2012). Does survey format influence selfdisclosure on sensitive question items? Computers in Human Behavior, 28, 251-256. doi:10.1016/j.chb.2011.09.007

Korte, J. (2012), Pieterse, M.E., Postel, M.G. \& Hoof, J.J. van (2012). Private peer group settings as an environmental determinant of alcohol use in Dutch adolescents: Results from a representative survey in the region of Twente. Health \& Place, 18, 892-897. doi:10.1016/j.healthplace.2012.02.010

Kuntsche, E., Kuntsche, S., Thrul, J., \& Gmel, G. (2017). Binge drinking: Health impact, prevalence, correlates and interventions. Psychology \& Health, 32, 976-1017. doi:10.1080/08870446.2017.1325889

Kuntsche, E., Rehm, J., \& Gmel, G. (2004). Characteristics of binge drinkers in Europe. Social Science \& Medicine, 59, 113-127. doi:10.1016/j.socscimed.2003.10.009 
Lammers, J., Kuntsche, E. N., Engels, R. C. M. E., Wiers, R. W. H. J., \& Kleinjan, M. (2013). Mediational relations of substance use risk profiles, alcohol-related outcomes, and drinking motives among young adolescents in the Netherlands. Drug and Alcohol Dependence, 133, 571-579. doi:10.1016/j.drugalcdep.2013.07.030

Licensing Act (2003). Retrieved from http://www.legislation.gov.uk/ukpga/2003/17/contents Local Alcohol Profiles for England (2017). Area profile - North East region. Retrieved from https://fingertips.phe.org.uk/profile/local-alcoholprofiles/data\#page/0/gid/1938132984/pat/6/par/E12000001/ati/102/are/E06000002/ii d/92904/age/173/sex/4

López-Caneda, E., Holguin, S., Cadaveira, F., Corral, M., \& Doallo, S. (2014). Impact of alcohol use on inhibitory control (and vice versa) during adolescence and young adulthood: A review. Alcohol and Alcoholism, 49, 173-181. doi:10.1093/alcalc/agt168 Loewenthal, K. M. (1996). An introduction to psychological tests and scales. London: UCL Press.

Lynam, D. R., \& Miller, J. D. (2004). Personality pathways to impulsive behavior and their relations to deviance: Results from three samples. Journal of Quantitative Criminology, 20, 319-341. doi:10.1007/s10940-004-5867-0

Malmberg, M., Overbeek, G., Monshouwer, K., Lammers, J., Vollebergh, W. A. M., \& Engels, R. C. M. E. (2010). Substance use risk profiles and associations with early substance use in adolescence. Journal of Behavioral Medicine, 33(6), 474-485. https://doi.org/10.1007/s10865-010-9278-4.

Malmberg, M., Kleinjan, M., Vermulst, A. A., Overbeek, G. J., Monshouwer, K., Lammers, J., \& Engels, R. C. M. E. (2012). Do substance use risk personality dimensions predict the onset of substance use in early adolescence? A variable- and person- 
centered approach. Journal of Youth and Adolescence, 41, 1512-1525.

doi:10.1007/s10964-012-9775-6.

Marks Woolfson, L., \& Maguire, L. (2010). Binge drinking in a sample of Scottish

undergraduate students. Journal of Youth Studies, 13, 647-659.

doi:10.1080/13676261003801804

McMahon, J., McAlaney, J., \& Edgar, F. (2007). Binge drinking behaviour, attitudes and beliefs in a UK community sample: An analysis by gender, age and deprivation.

Drugs: education, prevention and policy, 14, 289 - 303. doi:

$10.1080 / 09687630701288461$

Nibud (2011). Extra geld. Retrieved from http://www.nibud.nl/scholieren/inkomsten/extrageld.html

Norman, P., Armitage, C. J., \& Quigley, C. (2007). The theory of planned behavior and binge drinking: Assessing the impact of binge drinker prototypes. Addictive Behaviors, 32, 1753-1768. doi:10.1016/j.addbeh.2011.01.025

Norman, P., \& Conner, M. (2006). The theory of planned behaviour and binge drinking: Assessing the moderating role of past behaviour within the theory of planned behaviour. British Journal of Health Psychology, 11, 55-70.

doi:10.1348/135910705X43741

Norman, P., Conner, M. T., \& Stride, C. B. (2012). Reasons for binge drinking among undergraduate students: An application of behavioural reasoning theory. British Journal of Health Psychology, 17, 682-698. doi:10.1111/j.2044-8287.2012.02065.x

Office for National Statistics (2016). Adult drinking habits in Great Britain: 2005 to 2016. Retrieved from https://www.ons.gov.uk/peoplepopulationandcommunity/healthandsocialcare/drugus ealcoholandsmoking/bulletins/opinionsandlifestylesurveyadultdrinkinghabitsingreatbri 
tain/2005to2016\#: :text=In\%20Great\%20Britain\%2C\%2056.9\%25\%20of,on\%20their \%20heaviest\%20drinking\%20day.

O’Malley, P.M., Bachman, J.G., \& Johnston, L.D., (1983). Reliability and consistency in self-report of drug use. International Journal of the Addictions, 18, 805-824. doi:10.3109/10826088309033049

Overheid.nl (2013). Drank-en Horecawet. Retrieved from http://wetten.overheid.nl/BWBR0002458/geldigheidsdatum_01-03-2013.

Pirkle, E. C., \& Richter, L. (2006). Personality, attitudinal and behavioral risk profiles of young female binge drinkers and smokers. Journal of Adolescent Health, 38, 44-54. doi:10.1016/j.jadohealth.2004.09.012

Reich, R., Cummings, J., Greenbaum, P., Moltisanti, A., \& Goldman, M. (2015). The temporal 'pulse' of drinking: Tracking 5 years of binge drinking in emerging adults. Journal of Abnormal Psychology, 124, 635-647. doi:10.1037/abn0000061

Stautz, K., \& Cooper, A. (2013). Impulsivity-related personality traits and adolescent alcohol use: A meta-analytic review. Clinical Psychology Review, 33, 574-592. doi:10.1016/j.cpr.2013.03.003

Szmigin, I., Griffin, C., Mistral, W., Bengry-Howell, A., Weale, L., \& Hackely, C. (2008). Reframing 'binge drinking' as calculated hedonism: Empirical evidence from the UK. International Journal of Drug Policy, 19, 359 - 366. doi:

10.1016/j.drugpo.2007.08.009

van Wersch, A., \& Walker, W. (2009). Binge drinking as a social and cultural phenomenon: limitations of social-cognitive theories and the development of a grounded theoretical model. Journal of Health Psychology, 14, 124 - 134. doi:

10.1177/1359105308097952. 
Heavy Episodic Drinking in UK and Dutch Youths

Volksgezondheidenzorg.info (2018). Overmatige drinkers per wijk [excessive drinkers by ward]. Retrieved from

https://www.volksgezondheidenzorg.info/onderwerp/alcoholgebruik/regionaalinternationaal/regionaal\#node-overmatige-drinkers-wijk

Woicik, P. A., Stewart, S. H., Pihl, R. O., \& Conrod, P. J. (2009). The substance use risk profile scale: A scale measuring traits linked to reinforcement-specific substance use profiles. Addictive Behaviors, 34, 1042-1055. doi:10.1016/j.addbeh.2009.07.001.

World Health Organization [WHO] (2011). Global status report on alcohol and health. Switzerland: World Health Organization.

Zhao, X., Lynch Jr., J. G., \& Chen, Q. (2010). Reconsidering Baron and Kenny: Myths and truths about mediation analysis. Journal of Consumer Research, 37, 197-206. doi:10.1086/651 
Heavy Episodic Drinking in UK and Dutch Youths

Table 1

Descriptives of heavy drinking episodes by country of residence

\begin{tabular}{lccrrr}
\hline \multirow{2}{*}{ Country of residence } & \multicolumn{2}{c}{ Netherlands $(N=198)$} & \multicolumn{2}{l}{ United Kingdom $(N=95)$} & \multirow{2}{*}{$d$} \\
\cline { 2 - 5 } & Mean & SD & Mean & SD & \\
\hline Heavy-drinking episodes & 1.52 & 0.75 & 2.03 & 1.04 & -0.60 \\
Behavioral intention & 3.22 & 1.20 & 2.79 & 1.07 & 0.37 \\
Perceived behavioral control & 2.13 & 0.78 & 2.45 & 0.94 & -0.38 \\
Impulsivity & 2.23 & 0.43 & 2.19 & 0.50 & 0.10 \\
Sensation-seeking & 2.55 & 0.55 & 2.64 & 0.57 & -0.16 \\
\hline
\end{tabular}


Heavy Episodic Drinking in UK and Dutch Youths

Table 2

Correlations

\begin{tabular}{lrrrrr}
\hline & 1 & 2 & 3 & 4 & 5 \\
\hline 1 Heavy-drinking episodes & & & & & \\
2 Country of residence & 0.27 & & & & \\
3 Behavioral intention & -0.45 & -0.17 & & & \\
4 Perceived behavioral control & 0.42 & 0.18 & -0.59 & & \\
5 Impulsivity & 0.28 & -0.05 & -0.30 & 0.30 & \\
6 Sensation-seeking & 0.21 & 0.08 & -0.18 & 0.14 & 0.31 \\
\hline
\end{tabular}

Note. Pearson's correlations, except biserial correlations for variable country of residence. 
Table A1

Principal component analysis for impulsivity and sensation-seeking, pattern matrix

\begin{tabular}{|c|c|c|}
\hline & \multicolumn{2}{|c|}{ Component } \\
\hline & 1 & 2 \\
\hline I would like to skydive & 0.89 & 0.02 \\
\hline I enjoy new and exciting experiences even if they are unconventional & 0.69 & -0.06 \\
\hline I am interested in experience for its own sake even if it is illegal & 0.48 & 0.30 \\
\hline I would like to learn how to drive a motorcycle & 0.44 & 0.13 \\
\hline I would enjoy hiking long distances in wild and uninhabited territory & 0.41 & -0.15 \\
\hline I often don't think things through before I speak & -0.17 & 0.95 \\
\hline I usually act without stopping to think & 0.09 & 0.67 \\
\hline I often involve myself in situations that I later regret being involved in & 0.06 & 0.41 \\
\hline Generally, I am an impulsive person & 0.26 & 0.36 \\
\hline
\end{tabular}

Note. Cumulative variance explained: $50 \%$. Rotation: direct oblimin. 
Table A2

Principal component analysis for behavioral intention and perceived behavioral control, pattern matrix

\begin{tabular}{|c|c|c|}
\hline & \multicolumn{2}{|c|}{ Component } \\
\hline & 1 & 2 \\
\hline To me, drinking less than 6 units of alcohol on one night, when my friends drink more is ... & 0.83 & 0.05 \\
\hline To me, refusing an offered alcoholic drink when I actually have had enough is ... & 0.80 & 0.06 \\
\hline To me, drinking less than 6 units or more a week is ... & 0.70 & 0.03 \\
\hline To me, never drink 6 or more units in one occasion is ... & 0.69 & -0.21 \\
\hline To me, always drink less than my 'tax' is ... & 0.62 & -0.09 \\
\hline I intend to drink less than 6 units of alcohol per occasion & -0.03 & 0.90 \\
\hline I expect I'll drink less than 6 units of alcohol per occasion & -0.05 & 0.89 \\
\hline I intend never to exceed my 'tax' (so always drink less alcohol than your previously stated personal limit) & 0.04 & 0.77 \\
\hline
\end{tabular}

Note. Cumulative variance explained: $64 \%$. Rotation: direct oblimin: end-points 'easy' and 'hard'. 